ronalen Stammzellen. Im Tierexperiment ließ sich damit zudem die ZNS-Entzündung abschwächen. Erste klinische Studien sind in Planung. Noch weit von der klinischen Anwendung entfernt ist hingegen eine Behandlung mit induzierten pluripotenten Stammzellen, aus denen sich autologe Oligodendrozyten züchten lassen. Werden diese in die Gehirne neugeborener Mäuse injiziert, die aufgrund eines Gendefekts kein Myelin bilden konnten, kommt es zu einer großflächigen Myelinisierung und deutlichen Lebensverlängerung der Tiere, berichtete Lubetzki. Ein solcher Oligodendrozyten-Nachschub könnte gerade für ältere Patienten mit MS wichtig sein. Darauf deutet eine Untersuchung der Arbeitsgruppe um Dr. Sabine Pfeifenbring von der Universität Göttingen. Sie untersuchte Biopsien von Läsionen bei 29 Kindern sowie 24 Erwachsenen mit MS oder klinisch isoliertem Syndrom. In Läsionen mit erfolgreicher Remyelinisierung war die Oligodendrozytendichte bei Kindern verglichen mit der Umgebung nur um $13 \%$ reduziert, bei Erwachsenen um $31 \%$. „Bei Kindern erfolgt die Remyelinisierung schneller als bei Erwachsenen“, so Pfeifenbring. Erwachsene haben in den Läsionen auch weniger Oligodendrozyten-Vorläuferzellen. Offenbar nimmt deren Dichte mit dem Alter ab, und dies ist vermutlich ein Grund, weshalb die Remyelinisierung bei ihnen weniger gut klappt.

Thomas Müller, Springer Medizin

Parallel Session 9: Remyelination: mechanisms and therapeutic approaches. 32. ECTRIMS-Kongress, London, 14. - 17.9.2016

\title{
Remyelinisierung - nur geringer Erfolg mit Anti-LINGO-Antikörper
}

\author{
Der Anti-LINGO-Antikörper Opicinumab war in einer \\ Phase-II-Studie bei Patienten mit fortgeschrittener \\ MS Placebo kaum überlegen - wenn, dann nur in \\ mittlerer Dosierung sowie bei relativ geringer.
}

Es sind Ergebnisse, über die MS-Experten noch einige Zeit rätseln dürften: In der fünfarmigen Phase-II-Studie SYNERGY mit dem Antikörper Opicinumab profitierten am ehesten noch $\mathrm{Pa}$ tienten mit den beiden mittleren Dosierungen des neuen Präparats, nicht aber in der höchsten und niedrigsten - es deutete sich also eine inverse U-Kurve an. „Woran dies liegt, ist noch völlig unklar“, sagte Dr. Diego Cadavid vom Unternehmen Biogen, der die Resultate erstmals vorstellte. Die Forscher hatten sich von dem Antikörper eigentlich eine dosisabhängige klinische Verbesserung der MS-Symptome versprochen. Schließlich soll Opicinumab die Remyelinisierung vorantreiben und damit MS-bedingte Störungen zu einem gewissen Grad rückgängig machen - was natürlich nur gelingt, wenn die demyelinisierten Nervenfasern noch intakt sind.

Der Antikörper richtet sich gegen das Oberflächenglykoprotein LINGO-1, das die Myelinisierung aktiv hemmt. Wird das Protein außer Gefecht gesetzt, sollte dies die Myelinneubildung beschleunigen - wie im Tierversuchen gezeigt werden konnte. Auch die RENEW-Studie bei Patienten mit Optikusneuritis lieferte Hinweise auf einen Nutzen. Hier bestimmten die Ärzte die Unterschiede bei der Leitfähigkeit des Nervus opticus zwischen gesundem und betroffenem Auge, gemessen als Vollfeld visuell evoziertes Potenzial (FF-VEP). Nach etwa einem halben Jahr hatte sich die FF-VEP-Latenz bei doppelt so vielen Patienten mit der Anti-LINGO-1-Behandlung normalisiert wie mit Placebo (53\% vs. $26 \%$ ). Allerdings handelt es sich bei der Optikusneuritis um eine sehr frische Verletzung der schützenden Axonummantelung. Ob sich auch nach langjähriger MS-Erkrankung Fortschritte durch eine remyelinisierende Therapie erzielen lassen, sollte SYNERGY zeigen.

418 MS-Kranke erhielten über 72 Wochen hinweg Placebo oder alle vier Wochen eine Opicinumab-Infusion mit 3, 10, 30 oder 100 mg/kg Körpergewicht. Zusätzlich erhielten alle MSPatienten Interferon beta-1a. Die Patienten waren im Schnitt 40
Jahre alt und hatten einen medianen EDSS-Wert von drei Punkten, rund $20 \%$ waren an einer sekundär-progredienten MS erkrankt, bei allen mussten im Jahr vor der Studienaufnahme Hinweise auf eine bestehende Krankheitsaktivität aufgetreten sein. Die Forscher um Cadavid schauten nach Verbesserungen beim EDSS-Wert, beim 25-Fuß-Gehtest, beim Nine-Hole-PegTest (NHPT) und beim PASAT-3 (3-Second Paced Auditory Serial Addition Test). Der primäre Endpunkt wurde erreicht, wenn die Patienten über drei Monate hinweg eine EDSS-Verbesserung von mindestens einem Punkt erzielten und/oder sich in einem der anderen Tests um mindestens $15 \%$ verbessern konnten.

\section{Kein linearer Dosis-Wirkungs-Trend}

Wie sich zeigte, verbesserten sich jeweils $51 \%$ der Patienten unter Placebo sowie unter der 3-mg-Dosierung. Etwas höher war der Anteil mit 66\% und 69\% in den Gruppen mit $10 \mathrm{mg}$ und $30 \mathrm{mg}$ Opicinumab. Am schlechtesten schnitten jedoch die Patienten mit der höchsten Dosis ab - hier verbesserten sich nur $41 \%$, also noch weniger als unter Placebo. Signifikant im Vergleich zu Placebo war der Unterschied nur in der 30-mg-Gruppe. Das primäre Ziel, einen signifikanten linearen Dosis-Wirkungs-Trend zu beobachten, wurde verfehlt. Vielmehr deutete sich eine inverse U-Kurve an, die auch in den Einzelauswertungen zu sehen war. Die meisten Responder gab es in den 10- und 30-mg-Gruppen, die wenigsten mit $100 \mathrm{mg}$. Tendenziell schienen jüngere Patienten, solche mit schubförmiger MS und kurzer Krankheitsdauer sowie einer relativ geringen Hirnatrophie am ehesten zu profitieren. Für Cadavid war die ausgeprägte Placeboantwort ein großes Problem. „Wir hatten höchstens eine Responderrate von $40 \%$ erwartet." Ob und in welcher Weise das Studienprogramm mit Opicinumab fortgesetzt wird, ist noch unklar. Immerhin lege SYNERGY nahe, dass bestimmte Patientengruppen - jüngere und weniger schwer Erkrankte - von mittleren Dosierungen profitieren könnten. Dies werde wohl in künftigen Studien berücksichtigt, erläuterte Cadavid.

Thomas Müller, Springer Medizin

Parallel Session 9, Remyelination: mechanisms and therapeutic approaches. D. Cadavid: Efficacy analysis of opicinumab in relapsing multiple sclerosis: the Phase 2b SYNERGY trial. 32. ECTRIMS-Kongress, London, 14. - 17.9.2016 